\title{
Electron relaxation in a double quantum dot through two-phonon processes
}

\author{
V.N. Stavrou and Xuedong $\mathrm{Hu}$ \\ Department of Physics, State University of New York at Buffalo, New York 14260, USA
}

\begin{abstract}
We theoretically study the relaxation of electron orbital states of a double quantum dot system due to two-phonon processes. In particular, we calculate how the relaxation rates depend on the separation distance between the quantum dots, the strength of quantum dot confinement, and the lattice temperature. Enhancement of the rates by specific inter-dot distances and lattice temperatures, and the relative strength of different scattering channels are discussed. Our results show that although at low temperatures $(T \sim 1 \mathrm{~K})$ two-phonon processes are almost four orders of magnitude weaker compared to one-phonon processes in relaxing electron orbital states, at room temperature they are as important as one-phonon processes.

PACS numbers: 73.21.La, 71.38.-k, 03.67.Lx, 85.35.Be
\end{abstract}

\section{INTRODUCTION}

Semiconductor and superconductor nanostructure based quantum computing has attracted wide spread attention in recent years $\stackrel{1}{\underline{1}}$ Specifically, single electrons trapped in a semiconductor double quantum dot (QD) have been suggested as candidates of quantum bits (qubits), where the location of the electron in the two

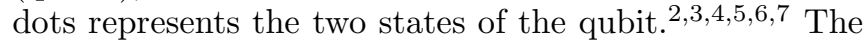
proposal of these so-called charge qubits has prompted careful experimental investigations ${ }^{8.9}$ during the past two years, which in turn motivated several detailed theoretical studies of the decoherence properties of these charge qubits $\stackrel{10,11,12,13}{ }$ So far all the calculations on electron relaxation due to electron-phonon interaction have focused on one-phonon processes, specifically single-phonon emission process. Here we calculate the relaxation rates due to two-phonon processes and investigate whether there exist any regime where two-phonon processes might be as important as one-phonon processes to charge qubit decoherence.

Two-phonon processes in a single three dimensional isotropic GaAs QD were studied in the context of electron relaxation in photoluminescence experiments more than

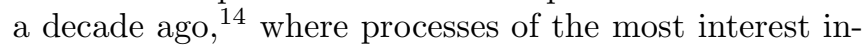
volve a longitudinal acoustic (LA) and a longitudinal optical (LO) phonon in the bulk phonon approximation ${ }^{15}$ Here we study the two-phonon processes in the context of relaxation of double dot charge qubits, where the qubit energy splitting $(\leq 1 \mathrm{meV})$ is much smaller than that studied before $(\geq 36 \mathrm{meV}$, when LO phonons are generally involved) so that our focus will be on two-acousticphonon processes. In these processes phonon density of state, qubit energy splitting, and lattice temperature together lead to interesting behaviors in the overall relaxation rates and relative strength of the different scattering channels.

The paper is organized as follows. In Section II we first briefly describe the electronic states we study and the electron-phonon coupling in our system. We then give the expressions for the two-phonon relaxation rates. In Section III we present our calculation results, showing how the relaxation rates depend on double dot parameters such as inter-dot distance and single dot confinement, and how these rates vary with temperature. We also discuss the physical mechanisms behind these obtained behaviors. In Section IV we draw some conclusions.

\section{THEORETICAL FORMALISM}

Our model system consists of two coupled QDs separated by a distance of $2 \alpha$, each of which is described by a two-dimensional (2D) harmonic well ${ }^{16}$ The singledot one-electron wavefunctions are 2D harmonic oscillator functions ${ }^{17.18}$ and are described in terms of the principal quantum number $n=0,1,2, \ldots$ and the angular momentum quantum number $m=0, \pm 1, \pm 2, \ldots$ as

$$
\psi_{\|}^{(n, m)}(\tilde{\rho}, \theta)=\sqrt{\frac{n !}{\pi l^{2}(n+|m|) !}} \tilde{\rho}^{|m|} e^{-\tilde{\rho}^{2} / 2} e^{i m \theta} \mathcal{L}_{n}^{|m|}\left(\tilde{\rho}^{2}\right)
$$

where $\mathcal{L}_{n}^{|m|}\left(\tilde{\rho}^{2}\right)$ are the Laguerre polynomials, and $\tilde{\rho}=$ $\left|\mathbf{r}_{\|}\right| / l$ is a scaled radius, with $l=\sqrt{\hbar / m^{*} \omega_{0}}$. The corresponding eigenvalues are

$$
E_{n m}=(2 n+|m|+1) \hbar \omega_{0} .
$$

Along the growth direction we assume an infinite quantum well (QW) confinement, so that the electron wavefunction has the form

$$
\psi_{z}(z)=\frac{1}{\sqrt{L_{z}}} \cos \left(\pi z / 2 L_{z}\right)
$$

For two QDs that are horizontally coupled, we use a simple in-plane confinement of two parabolic wells separated by an inter-dot distance $2 \alpha$ :

$$
V_{c}=\frac{1}{2} m^{*} \omega_{0}^{2} \min \left\{(x-\alpha)^{2}+y^{2}, \quad(x+\alpha)^{2}+y^{2}\right\} .
$$

The single electron wavefunction for the lateral direction can in general be expressed as a superposition of the 
single-dot wavefunctions:

$$
\left|\Psi_{\|}\right\rangle=\sum_{k} C_{k}\left|\psi_{\|, L}^{k}\right\rangle+D_{k}\left|\psi_{\|, R}^{k}\right\rangle,
$$

and the total wavefunction of the system of the coupled QDs is

$$
\Psi(\mathbf{r})=\Psi_{\|}\left(\mathbf{r}_{\|}\right) \psi_{z}(z)
$$

In the present study, the in-plane wavefunctions for the coupled-QD are calculated numerically by direct diagonalization, using reasonable parameters of a GaAs QW.

Electrons in GaAs interact with both acoustic and optical phonons. However, here we do not consider contributions from the optical phonons to the electron relaxation due to the small energy splitting in the double dot system we study. In this work, we calculate two-phonon relaxation rates caused by both deformation potential and piezoelectric interactions. The Hamiltonian that describes these interactions is given by:

$$
H=\sum_{\mathbf{q}}\left(\frac{\hbar}{2 \rho_{m} V \omega_{\mathbf{q}}}\right)^{1 / 2} \mathcal{M}(\mathbf{q}) \rho(\mathbf{q})\left(a_{\mathbf{q}}+a_{-\mathbf{q}}^{\dagger}\right),
$$

where $\rho_{m}$ is the mass density of the host material, $\omega_{\mathbf{q}}$ is the frequency of the phonon mode with wave vector $\mathbf{q}$, $V$ is the volume of the sample, $a_{\mathbf{q}}$ and $a_{-\mathbf{q}}^{\dagger}$ are phonon annihilation and creation operators, and $\rho(\mathbf{q})$ is the electron density operator. The interaction strength $\mathcal{M}(\mathbf{q})$ is defined by

$$
\mathcal{M}(\mathbf{q})=D|\mathbf{q}|+i \mathcal{M}_{\lambda}(\hat{q}),
$$

where the first term represents the deformation potential interaction with deformation constant $D$, and the second term, which is imaginary, describes the piezoelectric interaction. For zincblende crystals (e.g. GaAs), the piezoelectric term $\mathcal{M}_{\lambda}(\hat{q})$ takes the form

$$
\mathcal{M}_{\lambda}^{p z}(\hat{\mathbf{q}})=2 e e_{14}\left(\hat{q}_{x} \hat{q}_{y} \xi_{z}+\hat{q}_{y} \hat{q}_{z} \xi_{x}+\hat{q}_{x} \hat{q}_{z} \xi_{y}\right)
$$

Using second-order perturbation theory, scattering rates due to the emission and/or absorption of two LA phonons can be obtained

$$
\begin{aligned}
\Gamma_{++}= & \frac{\pi}{\hbar} \sum_{\mathbf{q}, \mathbf{k}}\left|\sum_{s}\left(\frac{M_{\mathbf{q}}^{i s} M_{\mathbf{k}}^{s f}}{E_{i}-E_{s}-E_{\mathbf{q}}}+\frac{M_{\mathbf{k}}^{i s} M_{\mathbf{q}}^{s f}}{E_{i}-E_{s}-E_{\mathbf{k}}}\right)\right|^{2} \\
& \times\left(N_{\mathbf{q}}+1\right)\left(N_{\mathbf{k}}+1\right) \delta\left(E_{i}-E_{f}-E_{\mathbf{q}}-E_{\mathbf{k}}\right) \\
\Gamma_{--}= & \frac{\pi}{\hbar} \sum_{\mathbf{q}, \mathbf{k}}\left|\sum_{s}\left(\frac{M_{\mathbf{q}}^{i s} M_{\mathbf{k}}^{s f}}{E_{i}-E_{s}+E_{\mathbf{q}}}+\frac{M_{\mathbf{k}}^{i s} M_{\mathbf{q}}^{s f}}{E_{i}-E_{s}+E_{\mathbf{k}}}\right)\right|^{2} \\
& \times N_{\mathbf{q}} N_{\mathbf{k}} \delta\left(E_{i}-E_{f}+E_{\mathbf{q}}+E_{\mathbf{k}}\right) \\
\Gamma_{+-}= & \frac{2 \pi}{\hbar} \sum_{\mathbf{q}, \mathbf{k}}\left|\sum_{s}\left(\frac{M_{\mathbf{q}}^{i s} M_{\mathbf{k}}^{s f}}{E_{i}-E_{s}-E_{\mathbf{q}}}+\frac{M_{\mathbf{k}}^{i s} M_{\mathbf{q}}^{s f}}{E_{i}-E_{s}+E_{\mathbf{k}}}\right)\right|^{2} \\
& \times N_{\mathbf{k}}\left(N_{\mathbf{q}}+1\right) \delta\left(E_{i}-E_{f}-E_{\mathbf{q}}+E_{\mathbf{k}}\right) .
\end{aligned}
$$

Here emission of two phonons (+LA+LA), absorption of two phonons (-LA-LA), and emission and absorption of one phonon each (+LA-LA, including both overall emission and absorption) are indicated by subscripts,++-- , and +- . The later two processes vanish at $T=0 \mathrm{~K}$. However, since experimental temperature is often in the same order as the qubit energy splitting, we include all two-phonon processes in our calculation. Indices $i$ and $f$ represent the initial and final electronic states, which are the ground and first excited double dot states respectively. Index $s$ refers to intermediate electronic states which are among the 13 higher energy states we include in the present calculation (the summation over s excludes the initial and final states, and the sum has converged). The matrix elements are calculated as in Ref. 13. Notice here that both deformation potential and piezoelectric interactions are included in the matrix element - they cannot be treated separately as in one-phonon processes. $N_{\mathbf{k}}$ is the Bose distribution function for the $\mathbf{k}$ phonon mode with energy $E_{\mathbf{k}}=\hbar \omega_{\mathbf{k}}$. The integrals over $\mathbf{k}$ and $\mathbf{q}$ in Eq. (10) to (12) are calculated by Monte Carlo code.

\section{RESULTS AND DISCUSSIONS}

At low temperatures, which are the operating temperature of the current generation charge-qubit-based architectures, two-phonon absorption processes should be much weaker than emission processes. Therefore we first evaluate the rates for an electron in the excited qubit state (the first excited state of the double dot as the initial state) to relax to the ground qubit state (final state) via two-phonon processes. Two channels are included here: (a) emission of two LA phonons (+LA+LA) and (b) emission of a high energy LA phonon and absorption of a lower energy LA phonon (+LA-LA). Throughout the paper the QW width is fixed at $2 L_{z}=10 \mathrm{~nm}$ and the material parameters are taken from Bruus et al..$^{22}$

In Figs. 1 and 2 we present the calculated relaxation rates due to the two overall emitting processes as functions of half inter-dot distance $\alpha$ and single dot confinement energy. For both figures the lattice temperature is fixed at $T=1 \mathrm{~K}$, a usual operating temperature for semiconductor charge qubits. The results presented in Fig. 1 have some interesting features. The most prominent is that the order of magnitude for both processes is much smaller compared to the one-phonon processes 13 . Therefore at low temperatures it should be sufficient to consider only the single-phonon processes when studying phonon-induced charge qubit decoherence.

Both curves in Fig. 10 display a clear peak at an intermediate inter-dot distance. This feature is an interplay between phonon density of state and the magnitude of the electron-phonon matrix element. When inter-dot distance increases, the qubit energy splitting decreases monotonically. This leads to a decreasing phonon density of state as it varies as $\omega^{2}$ as a function of phonon frequency. On the other hand, the magnitude of 


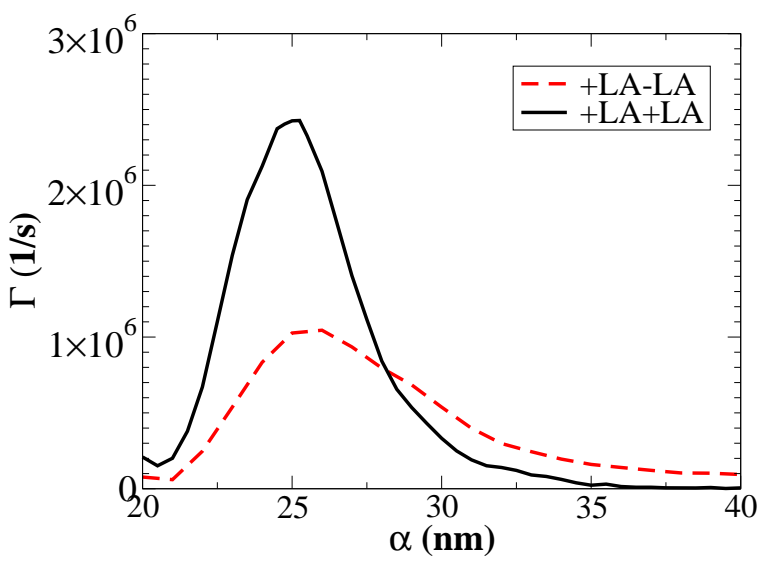

FIG. 1: Relaxation rates of an electron in a double dot due to two different two-phonon processes $(+\mathrm{LA}+\mathrm{LA}$ and $+\mathrm{LA}-\mathrm{LA})$ as functions of the half interdot distance $\alpha$. The confinement strength is $\hbar \omega=3 \mathrm{meV}$, lattice temperature $T=1 \mathrm{~K}$, and QW width $2 L_{z}=10 \mathrm{~nm}$. The oscillatory behavior of the $+\mathrm{LA}+\mathrm{LA}$ process at larger $\alpha$ is an artifact of the integration process.

electron-phonon matrix element increases with increasing $\alpha .13$ These two opposite trends dictate that a maximum should develop at an intermediate inter-dot distance for the electron relaxation rate.

One additional feature of Fig. 11 is the crossover between the two curves. The physics behind this crossover is even more complicated than the maxima of the two curves at an intermediate value of $\alpha$. Qualitatively, for $+\mathrm{LA}+\mathrm{LA}$ processes, both phonons involved have to have energies smaller than the qubit energy splitting, while for +LA-LA processes the emitted phonon has to have energy larger than the qubit energy splitting. As bulk acoustic phonons have a density of state proportional to $\omega^{2}$, the +LA-LA processes should be favored over +LA+LA processes. On the other hand, +LALA processes involve absorption of one phonon, which is thermally constrained so that its energy has to be relatively small (thus with a relatively small phonon density of state) compared to the thermal energy (in the current case $\sim 0.1 \mathrm{meV}$ ). This argument favors the $+\mathrm{LA}+\mathrm{LA}$ processes. Furthermore, we mentioned above that the electron-phonon interaction matrix element increases with increasing $\alpha$. Therefore, which process yields a higher relaxation rate is determined by considering all three factors of lattice temperature, electronphonon matrix element, and phonon density of state.

The results presented in Fig. 2 are obtained at a fixed interdot distance of $2 \alpha=50 \mathrm{~nm}$. Notice that here the maxima of the two relaxation rate curves are mostly determined by the qubit energy splitting (as shown in the inset) and the phonon density of state consideration. The matrix element plays a less important role because it is not as sensitive to the single-dot confinement en-

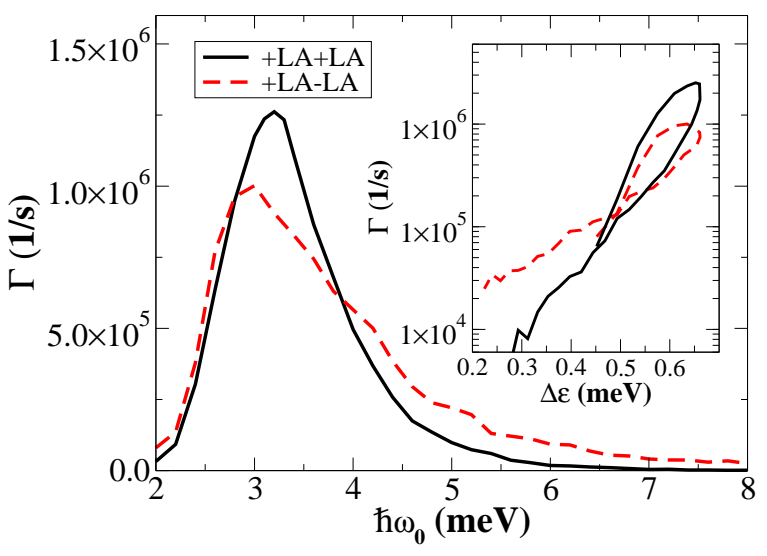

FIG. 2: Relaxation rates of an electron due to two different two-phonon (overall emission) processes $(+\mathrm{LA}+\mathrm{LA}$ and $+\mathrm{LA}-$ LA) as a function of the confinement strength. The rates versus the energy splitting $\Delta \varepsilon$ are shown in the inset. The half interdot distance is $\alpha=25 \mathrm{~nm}$, and the lattice temperature is $T=1 \mathrm{~K}$.

ergy as it is to inter-dot distance. Again the two rates have a crossover, with $+\mathrm{LA}+\mathrm{LA}$ processes faster at larger qubit energy splittings while +LA-LA processes faster for smaller qubit energy splittings. The physical mechanism is similar to that in Fig. 1]

In Fig. 3] we present the electron relaxation rates as functions of the lattice temperature for all the onephonon and two-phonon processes. At $T=1 \mathrm{~K}$, the strongest two two-phonon processes are the $+\mathrm{LA}+\mathrm{LA}$ and +LA-LA overall emission process, but the corresponding relaxation rates are more than three orders of magnitude smaller than the one-phonon emission process. The other two two-phonon processes are thermally suppressed because of the involvement of absorption of high energy phonon(s). On the other hand, at room temperature (corresponding to a thermal energy in the order of $30 \mathrm{meV}$, much larger than the qubit energy splitting of $<1 \mathrm{meV}$ ), the strongest two-phonon process, the +LALA (overall emission) process is only one order of magnitude smaller than the one-phonon processes (both emission and absorption). In other words, if the charge qubit states of a double quantum dot is to be used for some form of quantum information processing at high temperatures such as room temperature, two-phonon relaxation processes should also be taken into consideration.

At intermediate temperatures the relaxation rates have crossovers between the one-phonon-emission-onephonon-absorption processes and the two-phonon absorption or emission processes. The physical mechanism is similar to what we have discussed for Figs. 11 and 2 in that the thermal suppression of the higher energy phonons (which are only involved in +LA-LA and -LA+LA processes and have higher density of state) is gradually removed as temperature increases. At room 


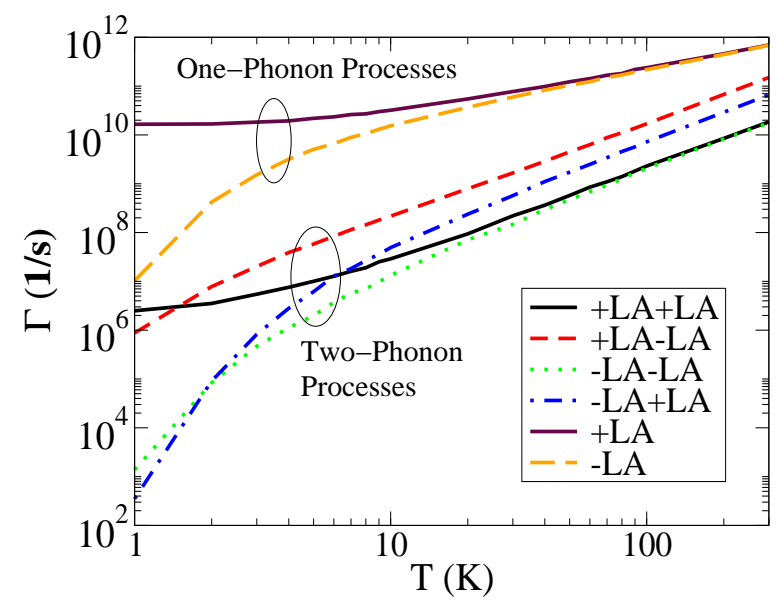

FIG. 3: Relaxation rates of an electron due to all the twophonon processes $[+\mathrm{LA}+\mathrm{LA},+\mathrm{LA}-\mathrm{LA}$ (overall emission), LA-LA (two-phonon absorption), and -LA+LA (overall absorption)] and one-phonon processes [+LA (one-phonon emission) and -LA (one-phonon absorption)] as functions of the lattice temperature. Here the confinement strength is $\hbar \omega=3$ $\mathrm{meV}$, and the half interdot distance is $\alpha=25 \mathrm{meV}$.

temperature the $+\mathrm{LA}+\mathrm{LA}$ and -LA-LA processes have almost the same relaxation rates because the thermal factors in Eqs. (10) and (11) are approximately the same for phonons with energy smaller than $1 \mathrm{meV}$ (recall that for two-phonon emission or absorption processes each of the phonons involved should have energy smaller than qubit energy splitting). Similarly, +LA-LA (overall emission) and $-\mathrm{LA}+\mathrm{LA}$ (overall absorption) processes also have similar rates at high temperatures. The discrepancy is mostly due to the involvement of higher energy phonons in these processes, for which the factors $N_{k}$ and $\left(N_{k}+1\right)$ could be sufficiently different.

\section{CONCLUSIONS}

In this study we have investigated two-acoustic-phonon processes (including both phonon emissions and absorptions) induced charge qubit relaxation in a semiconductor double quantum dot. Our results show interesting dependence of the relaxation rates on the lattice temperature and system configuration parameters such as inter-dot distance and single dot confinement energy. We have found that acoustic phonon density of state, electronphonon coupling matrix element, and phonon thermal distributions together lead to crossovers between different two-phonon scattering channels. In the context of charge qubit based quantum information processing, the two-phonon processes are much weaker than the onephonon processes at the relevant low temperatures, although their magnitudes are similar at the room temperature.

\section{ACKNOWLEDGMENT}

The work is supported in part by NSA and ARDA under ARO contract No. DAAD19-03-1-0128.
1 X. Hu and S. Das Sarma, Phys. Stat. Sol. (b) 238, 260 (2003); X. Hu, cond-mat/0411012 a brief review on quantum dot quantum computing.

2 A. Barenco, D. Deutsch, A. Ekert, and R. Jozsa, Phys. Rev. Lett. 74, 4083 (1995).

3 A. Ekert and R. Josza, Rev. Mod. Phys. 68, 733 (1996).

${ }^{4}$ M. S. Sherwin, A. Imamoglu, and T. Montroy, Phys. Rev. A 60, 3508 (1999).

5 T. Tanamoto, Phys. Rev. A 61, 022305 (2000).

6 A.A. Larionov, L.E. Fedichkin, and K.A. Valiev, Nanotechnology 12, 536 (2001).

7 L.C.L. Hollenberg, A.S. Dzurak, C. Wellard, A.R. Hamilton, D.J. Reilly, G. J. Milburn, and R.G. Clark, Phys. Rev. B 69, 113301 (2004).

8 T. Hayashi, T. Fujisawa, H.D. Cheong, Y.H. Jeong, and Y. Hirayama, Phys. Rev. Lett. 91, 226804 (2003).

9 J.R. Petta, A.C. Johnson, C.M. Marcus, M.P. Hanson, and A. C. Gossard, Phys. Rev. Lett. 93, 186802 (2004).

10 S.D. Barrett and G.J. Milburn, Phys. Rev. B 68, 155307 (2003).

11 L. Fedichkin and A. Fedorov, Phys. Rev. A 69, 032311 (2004).

12 S. Vorojtsov, E.R. Mucciolo, and H.U. Baranger, Phys. Rev. B 71, 205322 (2005).

13 V.N. Stavrou and X. Hu, cond-mat/0503481 Phys. Rev.
B 72 (in press).

14 T. Inoshita and H. Sakaki, Phys.Rev. B 46, 7260 (1992).

15 B. K. Ridley, Electrons and Phonons in Semiconductor (Cambridge University Press, 1996); N. C. Constantinou, J. Phys.: Condens. Mattter 3, 6859 (1991); V. N. Stavrou, M Babiker, and C. R. Bennett J. Phys.: Condens. Matter 13, 6489 (2001); V. N. Stavrou, C R Bennett, O.M.M. Al-Dossary, and M Babiker, Phys. Rev. B 63, 205304 (2001). V. N. Stavrou, Physica B-Condensed Matter 337, 87 (2003).

16 X. Hu and S. Das Sarma, Phys. Rev. A 61, 062301 (2000).

17 L. Jacak, P. Hawrylak, and A. Wójs, Quantum Dots (Springer, 1998).

18 U. Bockelmann Phys. Rev. B 50, 17271 (1994).

19 Gerald D. Mahan, Many-Particle Physics (Plenum Press, New York, 1990).

20 B. K. Ridley, Quantum processes in semiconductors (Clarendon Press, Oxford, 1982).

21 G. D. Mahan, in Polarons in Ionic Crystals and Polar Semiconductors, ed. J. T. Devreese (North-Holland, Amsterdam, 1972), pp.553-657.

${ }^{22}$ H. Bruus, K. Flensberg, and H. Smith Phys. Rev. B 48, 11144 (1993). 